\title{
Dual-Band Ultra-Wideband Bandpass Filter with Eight- Resonant Modes and Quad-Transmission Zeros Employing Synchronous-Quasi-Resonance
}

\author{
Jun-Ge LIANG, Cong WANG, Nam-Young KIM \\ RFIC Center, Kwangwoon University, 447-1 Wolgye-dong, Nowon-gu, Seoul 139-701, Korea \\ liangjun1991@hotmail.com, nykim@kw.ac.kr \\ Submitted December 12, 2017 / Accepted May 17, 2017
}

\begin{abstract}
This paper presented a dual-band ultra-wideband bandpass filter, which employs three sets of T-shape stubloaded resonators (TSSLRS). The new generation approaches of resonant mode (RM), which was named here as the synchronous-quasi-resonance (SQR), to generate more RMs were firstly presented and discussed. Five intrinsic RMs were generated by the even-/odd-mode resonances of the TSSLRS, while the other three additional RMS were achieved by the SQR among different TSSLRS. The fractional bandwidths of the dual-band filter were $42 \%$ (or $1.7 \mathrm{GHz}$ ) and $22 \%$ (or $1.1 \mathrm{GHz}$ ) with center frequencies of 3.45 and $5.10 \mathrm{GHz}$, respectively. The insertion loss of the filter was lower than $0.9 \mathrm{~dB}$, and the return loss was higher than $17 \mathrm{~dB}$. The frequency response of the filter was lower than $20 \mathrm{~dB}$ from $5.9 \mathrm{GHz}$ to $8.4 \mathrm{GHz}$. The high skirt selectivity and excellent out-of-band rejection were achieved by four controllable transmission zeros, which were generated by the virtual ground of the loaded-stub and the source-load coupling of the feed-lines. The proposed harmonic-suppressed dual-band ultra-wideband bandpass filter was fabricated on a Teflon substrate in order to verify the simulation results.
\end{abstract}

\section{Keywords}

Dual-band ultra-wideband, T-shape stub-loaded resonator, resonant mode, transmission zero, synchronous-quasi-resonance

\section{Introduction}

There is an increasing demand for dual-wideband (DWB) microwave devices for civilian use. Of particular interest are devices that support both the IEEE 802.16 WiMAX standard (3.5 GHz) and IEEE 802.11a WLAN standard $(5.1 \mathrm{GHz})$. After the US Federal Communications Commission authorized the unlicensed commercial application of ultra-wideband (UWB) communication systems, the technical specifications of the corresponding UWB bandpass filters (BPFs), namely low insertion loss (IL) and high out-of-band selectivity, have attracted a great deal of attention from researchers and engineers. Therefore, the dual-band ultra-wideband (DBUWB) filters that meet the UWB specification and support commonly-used citizens' dual-bands have promising application potential and high scientific research value; however, such designs are rarely found in the literature for the difficulty in building devices meeting both specifications of DWB and UWB. Herein, we firstly propose this DBUWB concept and implement this device with microstrip technique.

There are various design methodologies on UWB and DWB BPF designs respectively as references for the DBUWB design in this paper [1-12]. Either the UWB, DWB, or DBUWB's implementation mainly depends on the sufficient synchronization in the frequency response of both in-band and out-of-band. In terms of in-band frequency response, the low insertion loss, flat in-band characteristics, and wide-frequency-coverage passband are dependent on numerous of resonant modes (RMs) set at the specific frequency positions with high return loss (RL) values. As to the rejection on the out-of-band signal and harmonics, transmission zeros (TZs) work as the key elements. There are generally two schemes for constructing multiple RMs for UWB, namely via the multi-mode resonator and hybrid-resonator [1], [2]. In [1], it is mentioned that an UWB BPF can be realized using the high order resonances of a multi-mode resonator. However, the mutual effects of different RMs sourced from one resonator conduce a high design difficulty. An alternative to this scheme is to utilize a hybrid resonator structure, such as the parallel-coupled stepped-impedance resonator (SIR) presented in [2], which can also ameliorate the design flexibility. Besides, there are also various methods in implementing DWB BPF [3-12]. First, a cascade connection of a BPF followed by a bandstop filter, in which the latter filter is used to divide the passband into two separate bands, namely dual-band, can be considered as an effective method [3]. An alternative solution is to introduce TZs instead of a stopband to divide the passband [4]. Moreover, another type design takes advantage of the second harmonic in order to generate a dual-band, such as applying SIRs [5] or stub-loaded resonators [6]. Moreover, a DWB BPF can also be realized by combining multi-set resonators with common inputs and outputs, which is the same way as hybrid-resonator structure in UWB design [7]. 
The DBUWB BPF presented in this article incorporates several improvements in terms of the newly presented design methodologies and operating mechanisms. First, extra three RMs are generated due to the synchronousquasi-resonance (SQR), which is firstly proposed to improve the in-band flatness and expand the passband width with the identical structure of the normal even-odd-mode scheme. Additionally, for the first time, the concept of UWB and DWB is combined into high-performance DBUWB design meeting both their specifications. Lastly, the TZs generated by the virtual ground effect of the loaded-stubs and the source-load coupling (SLC) result in both strict frequency selectivity and excellent rejectionband performance [8-10].

The constitution of the BPF is analyzed based on a general prototype in the form of admittance model proposed in Sec. 2, which can constitute the DBUWB BPF in a modular scheme to meet both the specifications of UWB and DWB. The prototype for realizing triple-band BPF is developed with its network model to elaborate the voltagecurrent relation. Then, in Sec. 3, the corresponding tripleband BPF layout is depicted and RMs/TZs generation principles are analyzed to validate the above prototype. Furthermore, in Sec. 4, a method for merging three passbands of previous triple-band BPF into a single DBUWB is described in detail. The operation mechanism of RMs and TZs is separately discussed in Sec. 5 employing current distribution plot in resonant frequencies and frequency response variations caused by resonator's length change. Finally, in Sec. 6, the frequency response predicted by the simulation is validated by measuring the performance of the filter fabricated on Teflon substrate with $\varepsilon_{\mathrm{r}}=2.54$, $\tan \delta=0.002$ and thickness $0.54 \mathrm{~mm}$.

\section{Configuration}

\subsection{General Admittance Model}

The essence of filter design is to develop a circuit to generate enough RMs and TZs in necessary and arrange their positions in frequency response. The odd-/even-mode principle was considered as a way to generate RMs and Jinverter was usually used to introduce the attenuation poles. Thus, an admittance model was presented in Fig. 1(a) with detail electrical length and admittance for transmission line and J-inverters. The lossless transmission network could be described in a set of ABCD parameters to represent the current and voltage relationship. The subnetwork $N_{11}$, which was the network of the transmission lines inside the blue dash line, could be given with impedance $Z=1 / Y$ as

$$
\left[\begin{array}{ll}
A_{11} & B_{11} \\
C_{11} & D_{11}
\end{array}\right]=\left[\begin{array}{cc}
\cos \left(2 \theta_{11}\right) & \mathrm{j} Z / 2 \sin \left(2 \theta_{11}\right) \\
2 \mathrm{j} Y \sin \left(2 \theta_{11}\right) & \cos \left(2 \theta_{11}\right)
\end{array}\right] .
$$

Each of J-inverter gives a phase shift of \pm 90 degrees or an odd multiple, thereof the ideal admittance inverters have the ABCD matrix

$$
\left[\begin{array}{ll}
A_{J} & B_{J} \\
C_{J} & D_{J}
\end{array}\right]=\left[\begin{array}{cc}
0 & \pm 1 / \mathrm{j} J \\
\mp \mathrm{j} J & 0
\end{array}\right] \text {. }
$$

The sub-network $N_{12}$ is the stub-loaded structure in the middle of $N_{11}$, which shorts the $N_{11}$ to the ground. Its ABCD parameters can be summarized as below

$$
\begin{aligned}
& {\left[\begin{array}{ll}
A_{12} & B_{12} \\
C_{12} & D_{12}
\end{array}\right]=} \\
& {\left[\begin{array}{cc}
\frac{\sin \left(\theta_{12}+\theta_{13}\right)+\sin \theta_{12}\left(\cos \theta_{13}-2\right)}{\sin \theta_{13}} & \mathrm{j} Z\left(\sin \theta_{12}+\cos \theta_{12}\right) \\
\mathrm{j} Y \frac{\cos \left(\theta_{12}+\theta_{13}\right)+\cos \theta_{12}\left(\cos \theta_{13}-2\right)}{\sin \theta_{13}} & \cos \theta_{12}-\sin \theta_{12}
\end{array}\right]}
\end{aligned}
$$

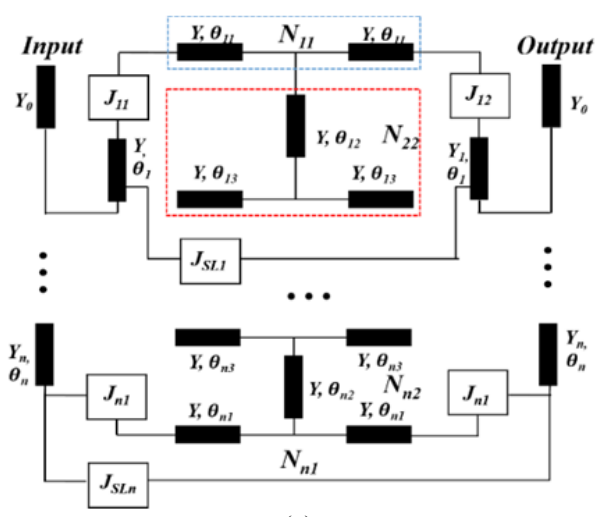

(a)

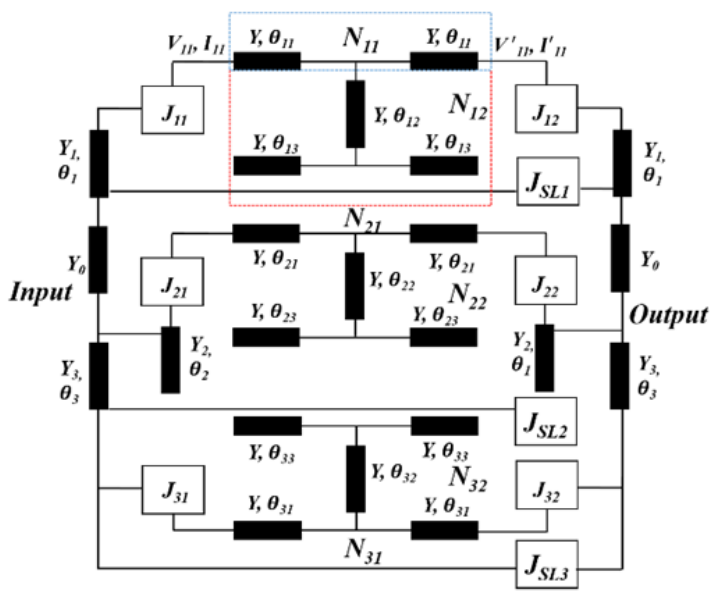

(b)

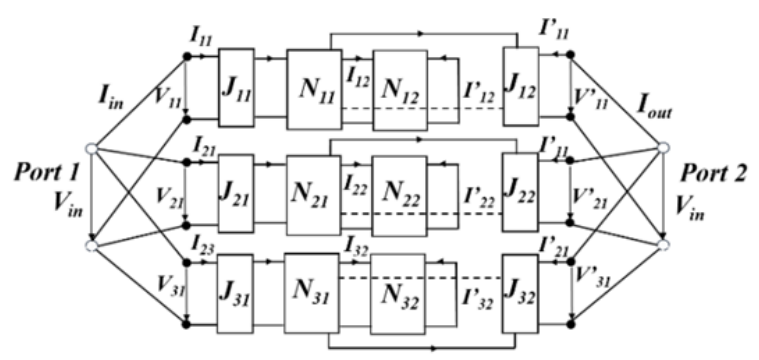

(c)

Fig. 1. (a) General admittance model for building BPF, (b) admittance model for implementing DBUWB BPF, (c) equivalent network of DBUWB BPF with voltage and current relationship. 


\subsection{Admittance Model and Network for Triple-band BPF}

Each group of the transmission line and loaded-stub, i.e., $N_{11}$ and $N_{12}$, corporates and forms one passband. A triple-band BPF admittance model and its equivalent network were shown in Fig. 1(b) and (c) respectively. In terms of the internal current relationship of this device, the overall circuit based on ABCD parameters could be calculated. Thus, the reflection coefficient, $S_{11}$ can be transferred by the ABCD parameters, as

$$
S_{11}=\left(A+\frac{B}{Z_{0}}-C Z_{0}-D\right) /\left(A+\frac{B}{Z_{0}}+C Z_{0}+D\right)
$$

where the terminal impedance $Z_{0}=50 \Omega$, and the RM generates at the condition where $\left|S_{11}\right|=0$, which means

$$
A+B / Z_{0}-C Z_{0}-D=0 \text {. }
$$

This formula provides the necessary and sufficient conditions of RM generation and contains the variables of admittance and electrical length for all resonators involved.

\section{Layout Configuration}

\subsection{Corresponding Layout}

The layout of the proposed DBUWB BPF was shown in Fig. 2(a), and the individual components that made up the complete design were shown separately in Figs. 2(b), (c), and (d), and were denoted T-shape stub-loaded resonator I (TSSLR I), TSSLR II, and TSSLR III. The signal transmission in the proposed BPF was realized by the coupling between the feed-lines and resonators. In other words, the in-band frequency response performance was largely influenced by the quality of the coupling. The coupling coefficients $k$ increased as the overlap between the coupling lengths among the feed-lines and resonators increased, the gap distances decreased, and as the strip-lines involved in the coupling were narrowed [11].

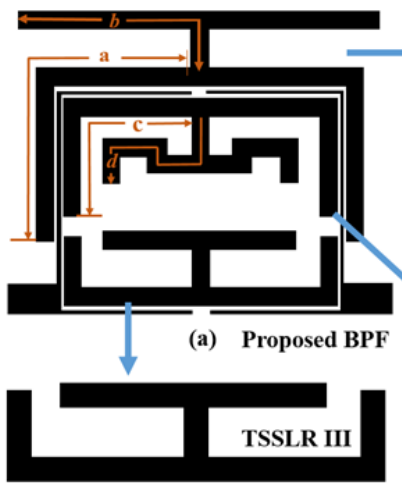

(c)

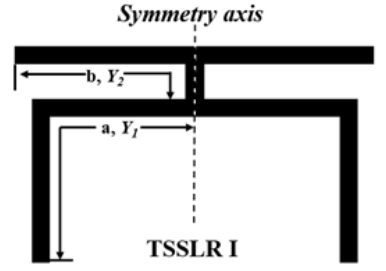

(b)

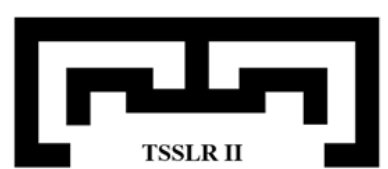

(d)
Fig. 2. (a) Layout of the proposed DBUWB BPF. (b) Upper resonator indicated as TSSLR I. (c) Lower resonator indicated as TSSLR III. (d) Middle resonator indicated as TSSLR II.
In the proposed filter, the width of the input/output ports was $1.5 \mathrm{~mm}$ in order to achieve a $50 \Omega$ impedance match to reduce the reflection loss. Besides, the feed-lines were designed to surround TSSLR II and TSSLR III, while TSSLR I was located above the upper feed-lines. The feedlines for the odd-modes in the three TSSLRs were fully coupled to maximize the overlap coupling length. Meanwhile, the gap distance between the resonators and feedlines as well as the widths of feed-lines were designed to be $0.2 \mathrm{~mm}$, which approached the highest fabrication precision possible on the Teflon substrate. With the special structures discussed above, the very high $k$ realized between the feed-lines and resonators led to a reduction of the in-band IL.

\subsection{RM Generations}

The proposed work was developed from a TSSLR with two controllable RMs and one TZ. As shown in Fig. 2(b), TSSLR I was composed of a conventional $\lambda / 4$ microstrip resonator and a loaded-stub. This structure could be analyzed using even-/odd-mode theory because the whole structure was symmetrical. The length of the resonator was $2 a$, and $2 Y_{1}$ denoted its characteristics admittance. Hence, the electric length was $2 \theta_{1}=2 \beta a$, where $\beta$ was the propagation coefficient. The end of the loaded-stub was designed using a bifurcation to reduce the overall size. The length of the loaded-stub was $2 b$, hence the electric length $2 \theta_{2}=2 \beta b$, and $2 Y_{2}$ represented the characteristic admittance. Thus, RMs generation sources could be classified as two categories as follows:

\section{Case 1: Even-lodd-mode}

When considering odd-mode excitation, the bisection of the $\lambda / 2$ resonator behaved as a short-circuited resonator, as shown in the lower part of Fig. 3(a). The input admittance for the odd-mode could be expressed as:

$$
Y_{\text {odd }}=\frac{Y_{1}}{\mathrm{j} \tan \theta_{1}}
$$

therefore, $Y_{\text {odd }}=0$ under the resonant condition, and the odd-mode frequency could be calculated as:

$$
f_{\text {odd }}=\frac{(2 n-1) c}{4 a \sqrt{\varepsilon_{\text {eff }}}}
$$

where $n=1,2,3 \ldots$, and $c$ was the speed of light in free

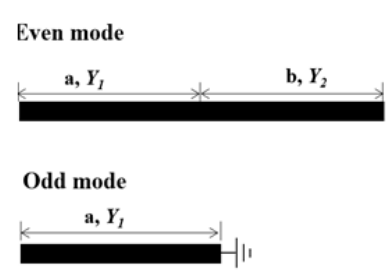

(a)

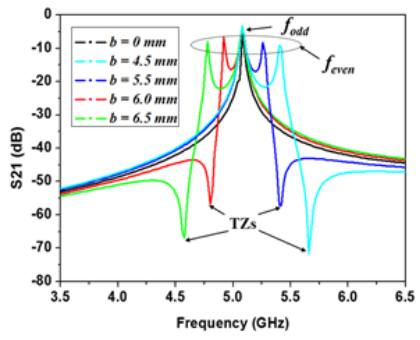

(b)
Fig. 3. (a) Even- and odd-mode equivalent circuits of TSSLR I, and (b) $\mathrm{S}_{21}$-variations of the TSSLR I with different lengths of $b$ 
space. The term $\varepsilon_{\text {eff }}$ represented the effective dielectric constant of the substrate, and was equal to 2.54 in this case.

For even-mode excitation, the half bisection behaved as an open-circuited $\lambda / 2$ resonator, as shown in Fig. 3(a). Hence, the input admittance for even-mode is given as

$$
Y_{\text {even }}=\mathrm{j} Y_{1} \frac{2 Y_{1} \tan \theta_{1}+Y_{2} \tan \theta_{2}}{2 Y_{1}-Y_{2} \mathrm{j} \tan \theta_{1} \tan \theta_{2}}
$$

under resonant conditions, $Y_{\text {even }}=0$. Thus, the even-mode frequency could be calculated as

$$
f_{\text {even }}=\frac{n c}{2(a+b) \sqrt{\varepsilon_{\text {eff }}}}
$$

where $n, c$, and $\varepsilon_{\text {eff }}$ were the same as described above. It could be observed from (2) and (4) that the even-mode resonant frequency was determined by the length of both the $\lambda / 2$ resonator and loaded-stub, while the odd-mode was only controlled by the length of the $\lambda / 2$ resonator.

The simulated $S_{21}$-variation of TSSLR I was plotted in Fig. 3(b) based on different lengths of even-mode $b$. Only one resonant mode was generated at $5.25 \mathrm{GHz}$ when the loaded-stub was removed, which was referred to as the odd-mode. If the loaded-stub in the middle section of the odd-mode was shunted and the length increased from $4.5 \mathrm{~mm}$ to $6.5 \mathrm{~mm}$, then an even-mode resonance emerged and shifted from $5.50 \mathrm{GHz}$ to $4.75 \mathrm{GHz}$ while the oddmode remained unchanged. A TZ was generated and moved along with the even-mode as it shifted during this process. In addition, the RL of even-mode was always lower than the RL of odd-mode as a result of their different coupling strengths with the feed lines.

\section{Case 2: Synchronous-Quasi-Resonance}

Among the passband interval, the resonant state of resonators was varied in dynamic. Their maximum resonances were discussed in even-/odd-mode cases. Herein, a hypothesis was given that different resonators can combine in their dynamic-resonant status to form a new RM named as SQR. In addition, SQR could only occur on resonators owning similar electrical lengths, which could introduce dynamic-resonance in the same frequency. However, there was no interference between the odd- and even-mode in one resonator based on a theory of asynchronously tuned coupled resonators. Hence, only two resonators located in disparate sections could meet the basic conditions of SQR.

\subsection{TZs Generations}

Signal transmission in the proposed filter was based on the multi-path coupling. In addition to the coupling path constructed by TSSLRs, when the feed-lines are sufficiently close, a new path will generate by SLC. The essential role for feed-lines in BPF was to input or output the microwave energy for resonator's inside. The largest overlap length and the nearest distance with resonators, as well as the narrowest widths of feed-lines ensured the energy concentration and avoided the loss. However, all microstrip

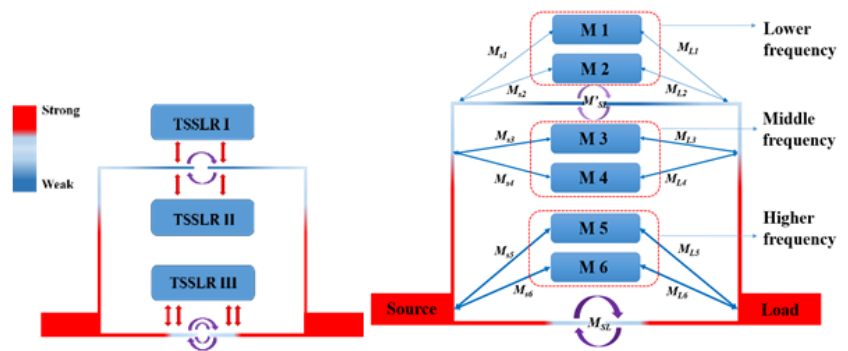

(a)

(b)

Fig. 4. (a) Coupling scheme between the proposed feed-lines and TSSLRs. (b) Detailed coupling topology of the proposed DBUWB BPF.

structures suffered from inevitable losses, including conductor loss, substrate loss, and radiation loss. Therefore, as shown by the colors in Fig. 4(a), the current density decreased with the increased-distance from the input/output ports. The closest resonator to the input/output ports was TSSLR III, which led to a very strong $k$ value. In addition, the strength of the SLC between the lower feed-lines was high due to the close coupling distance and the narrowness of the strip-lines. Thus, the $k$ value relationship between the resonators was TSSLR III > TSSLR II > TSSLT I, and the SLC between the lower feed-lines was stronger than the SLC between the upper feed-lines. In Fig. 4(a), different symbols were used to represent the varied $k$ value between the feed-lines and resonators, as well as the strength of the SLCs.

In Fig. 4(b), the different thickness used to represent the coupling lines between the resonators and feed-lines were also used to graphically express different $k$ values. The basic rule that applied here was that $k$ went down when far from the source/load, and was higher in odd-mode than in even-mode due to the direct coupling between the oddmode and feed-lines. The odd-mode and even-mode of TSSLR I were expressed as M2 and M1, respectively, while M3, M4, M5, and M6 used to represent the odd/even modes for TSSLR II and TSSLR III. Thus, the transmission matrix based on $k$ could be expressed as [13]:

$$
\mathbf{M}_{i j}=\left[\begin{array}{cccccc}
0 & M_{\mathrm{S} 1} & M_{\mathrm{S} 2} & \cdots & M_{\mathrm{S} 6} & M_{\mathrm{SL}} \\
M_{1 \mathrm{~S}} & M_{11} & 0 & \cdots & 0 & M_{\mathrm{S} 1} \\
M_{2 \mathrm{~S}} & 0 & M_{22} & \cdots & 0 & M_{\mathrm{S} 1} \\
\vdots & \vdots & \vdots & \ddots & \vdots & \vdots \\
M_{6 \mathrm{~S}} & 0 & 0 & \cdots & M_{66} & M_{S 1} \\
M_{\mathrm{LS}} & M_{\mathrm{L} 1} & M_{\mathrm{L} 2} & \cdots & M_{\mathrm{L} 6} & 0
\end{array}\right] .
$$

For the proposed structure to be symmetric, $k$ must satisfy the relationship that $M_{\mathrm{S} 1}=-M_{1 \mathrm{~L}}, M_{\mathrm{S} 2}=-M_{2 \mathrm{~L}}$, $M_{\mathrm{S} 3}=-M_{3 \mathrm{~L}}, M_{\mathrm{S} 4}=-M_{4 \mathrm{~L}}, M_{\mathrm{S} 5}=-M_{5 \mathrm{~L}}$, and $M_{\mathrm{S} 6}=-M_{6 \mathrm{~L}}$. The mathematical equations for the TZs could be expressed as follows:

$$
\begin{gathered}
T Z_{1}=\frac{\left(M_{11} M_{\mathrm{S} 2}^{2}-M_{22} M_{\mathrm{S} 1}^{2}\right)}{\left(M_{\mathrm{S} 2}^{2}-M_{\mathrm{S} 1}^{2}\right)}, \\
T Z_{2}=\frac{\left(M_{33} M_{\mathrm{S} 4}^{2}-M_{44} M_{\mathrm{S} 3}^{2}\right)}{\left(M_{\mathrm{S} 4}^{2}-M_{\mathrm{S} 3}^{2}\right)},
\end{gathered}
$$




$$
T Z_{3}=\frac{\left(M_{55} M_{\mathrm{S} 6}^{2}-M_{66} M_{\mathrm{S} 5}^{2}\right)}{\left(M_{\mathrm{S} 6}^{2}-M_{\mathrm{S} 5}^{2}\right)} .
$$

Since the $k$ of even-mode were always lower than odd-mode, such as, $M_{S 1}<M_{\mathrm{S} 2}, M_{\mathrm{S} 3}<M_{\mathrm{S} 4}$ and $M_{\mathrm{S} 5}<M_{\mathrm{S} 6}$, at least three TZs would be generated at finite frequencies, which were related to even-/odd-mode was analyzed in Fig. 3(b). In short, the $k$ values between different elements should meet the relationship that $M_{\mathrm{S} 1}<M_{\mathrm{S} 2}<\ldots<M_{\mathrm{S} 6}$ and $M_{\mathrm{SL}}^{\prime}<M_{\mathrm{SL}}$.

\section{DBUWB Design Process}

\subsection{Triple-band BPF Design Example}

The line styles denoted as $a, b, c$, and $d$ were used to indicate the lengths of the odd-/even-modes of TSSLR I and TSSLR II in Figs. 2(a) and Fig. 5. Fig. 5 showed the specific dimensions in different steps during the design process and illustrated four main adjustment procedures in order to clearly explain the operation on TSSLR I and TSSLR II. Four procedures were shown along the horizontal axis in Fig. 5, while the corresponding physical length adjustments for the four resonators were shown on the vertical axis.

The first step in the process was to establish a tripleband BPF, as shown in Fig. 6(a). The parameters were set according to Process 1 (P1) in Fig. 5. The length of the even-mode of the TSSLR was made shorter than the oddmode in order to make sure that both the even-mode and $\mathrm{TZ}$ emerge at the right of the odd-mode in the frequency response. In addition, the dimension relationship between the three TSSLRs was set to be TSSLR I > TSSLR II > TSSLR III. Hence, they could be configured to operate in different frequency ranges, and thereby achieved a tripleband BPF with three TZs, as shown in Fig. 6(a). The shadows in Fig. 6(a) were used to mark the passbands. The third passband at $5 \mathrm{GHz}$ was realized by TSSLR III with a lower in-band IL and wider bandwidth than the other two bands. The first passband generated by TSSLR I had the narrowest bandwidth and highest IL among three bands. The reason behind the differing in-band performance of the various bands was a consequence of the different $k$ value of three TSSLRs, as analyzed above. Additionally, three TZs were

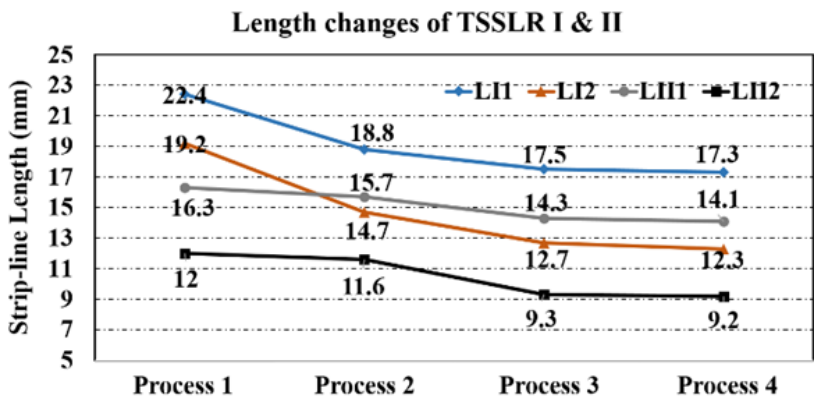

Fig. 5. Length changes of TSSLR I \& II in different steps during the design process.

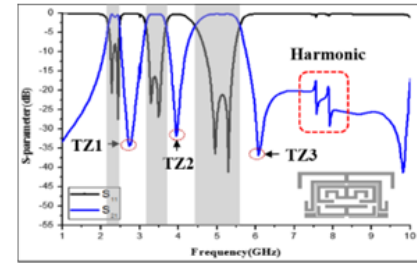

(a)

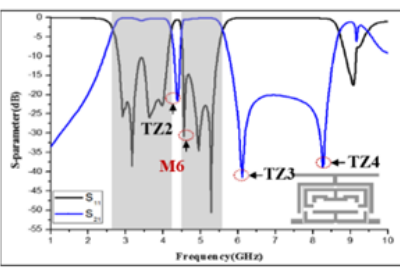

(c)

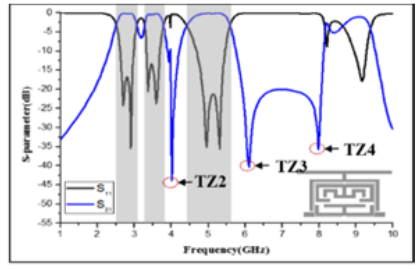

(b)

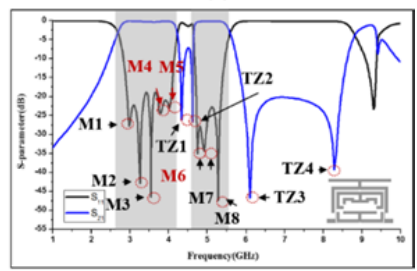

Fig. 6. Frequency responses of the proposed BPF during the design process: (a) Process 1 (P1): triple-passbands BPF with six RMs and three TZs. (b) Process 2 (P2): middle process BPF with six intrinsic RMs and three TZs. (c) Process 3 (P3): merged DBUWB BPF with seven RMs and three TZs. (d) Process 4 (P4): final DBUWB BPF with eight RMs and four TZs.

generated to increase the selectivity of the skirt, and two harmonic modes were generated at approximately $8 \mathrm{GHz}$.

In order to realize a DBUWB, the basic procedure was to divide the second passband and then move the RMs into the first and third passbands, respectively. In this way, both the first and third passbands could be widened and the in-band performance improved due to the increased number of RMs. The practical design process of varying the resonator lengths was plotted in Fig. 5 described as four steps: P1, P2, P3, and P4.

\subsection{Merging Passbands}

In step $\mathrm{P} 2$, the lengths of $a, b, c$, and $d$ were shortened. The ranges of variation for $a$ and $b$ were larger than those for $c$ and $d$ in order to move the first passband closer to the second passband. As shown in Fig. 6(b), TZ1, which was located in the middle of the first and second passbands in P1 vanished in this step, and both of the passbands tended to blend together. Meanwhile, the new TZ (TZ4) generated by the SLC between the lower feedlines emerged at $8.0 \mathrm{GHz}$. In fact, TZ4 should exist in the triple-band BPF of P1. However, the influence of the second harmonic at $8 \mathrm{GHz}$ forced it to disappear. Moreover, the length of $c$ was slightly longer than $b$ in this step and caused their electric lengths to be similar.

In step P3, the lengths of $a, b, c$, and $d$ were decreased by similar amounts in order to further merge the first two passbands and simultaneously shift the even-mode of TSSLR II to a higher frequency. As shown in Fig. 6(c), this step caused TZ1 to disappear completely and an UWB formed at the center frequency of $3.5 \mathrm{GHz}$. A new RM was generated at $4.6 \mathrm{GHz}$ by the even-mode of TSSLR II, which could be controlled by $b$ to further widen and improve the in-band performance of the second passband. However, the RL at the center frequency of the first passband is $11 \mathrm{~dB}$, which was still not high enough to ensure 
a good in-band performance and warranted further optimization in the next step.

Finally, in step P4, the lengths of $a, b, c, d$ were modified slightly to further improve the final performance. The frequency response was shown in Fig. 6(d), and consisted of five RMs in the first band and three RMs in the second band, indicated as M1, M2 ..., and M8. The two TZs located in the center of the two passbands were denoted TZ1 and TZ2, and two TZs in the upper stopband were denoted as TZ3 and TZ4.

\section{Operation Mechanism}

\subsection{Resonant Modes}

To analyze the mechanism of operation of the eight RMs in the frequency response, the current distribution of the resonant frequencies were plotted using Sonnet (Sonnet Software Inc.), and were shown in Fig. 7, in which, M1 and M2 were the odd-mode and even-mode of TSSLR I, respectively, shifting from approximately $2.3 \mathrm{GHz}$ in $\mathrm{P} 1$ to $2.98 \mathrm{GHz}$ and the $3.20 \mathrm{GHz}$ as a result of the length shortening of $a$ and $b$ during the design process. Modes M3 and M4 at $3.55 \mathrm{GHz}$ and $3.74 \mathrm{GHz}$, respectively, were generated by the SQR of the even-mode of TSSLR I and oddmode of TSSLR II. As mentioned above, the length of $c$ was slightly longer than $b$ after step P2, and the similar electric lengths caused SQR at the same frequency. However, the current density of each resonator in the case of SQR was not as high as their inherent RM. In the current distribution of M3, the even-mode strength of TSSLR I was higher than that in the odd-mode of TSSLR II, while it was reversed in the current distribution of M4. Mode M5 was the even-mode of TSSLR II and was generated at $4.04 \mathrm{GHz}$. All of the above mentioned five RMs operated together to ensure the good in-band performance of the first passband. In the second passband, modes M6 and M7 were generated at $4.70 \mathrm{GHz}$ and $4.86 \mathrm{GHz}$, respectively, due to SQR constructed by both the even-mode of TSSLR II and odd-mode of TSSLR III. Mode M8 generated at $5.30 \mathrm{GHz}$ by the even-mode of TSSLR II formed the second UWB together with M6 and M7.

\section{UWB in lower frequency}

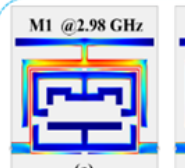

(a)

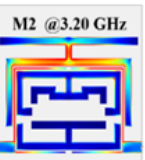
(b)
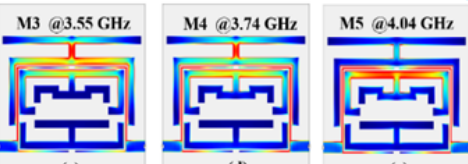

(d)

UWB in higher frequency

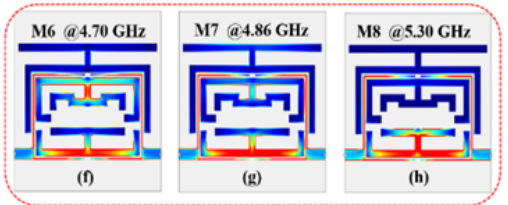

Fig. 7. Simulated current distributions at the resonant frequencies of UWB BPF.

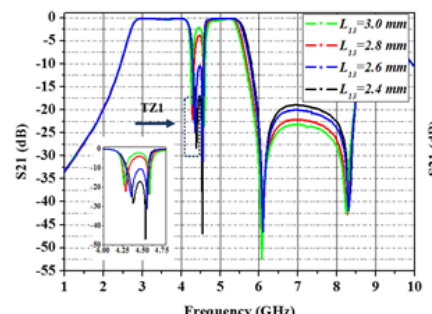

(a)

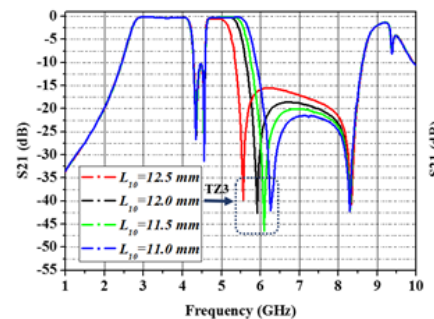

(c)

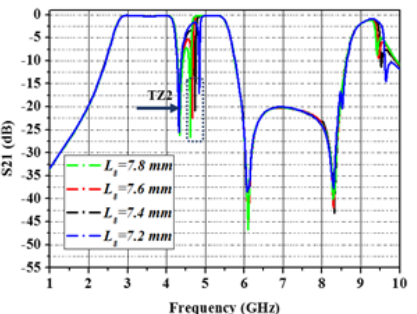

(b)

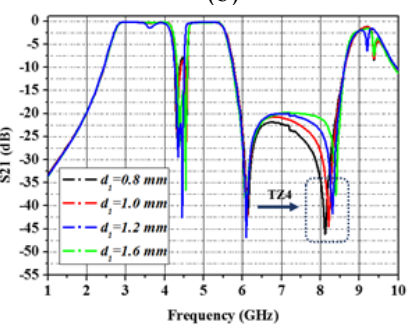

(d)
Fig. 8. Variations in the frequency response by changing: (a) $L_{13}$ of TSSLR III, (b) $L_{8}$ of TSSLR II, (c) $L_{10}$ of TSSLR III, and (d) $d_{1}$ of feed-lines.

\subsection{Transmission Zeros}

In addition to the excellent in-band performance ensured by the presence of eight RMs, a high degree of outof-band rejection and skirt selectivity was also achieved by the presence of four TZs. The variations in the frequency response caused by changes in the physical dimensions were plotted in Fig. 8. TZ1 shifted to a higher frequency when the length of $L_{13}$ in Fig. 9 decreased, as shown in Fig. 8(a), which implied that the odd-mode of TSSLR III controlled the position of TZ1. The change in TZ1 modified both the selectivity and bandwidth of the first passband. Moreover, when the length of $L_{8}$ decreased, then TZ2 shifted to a higher frequency while the location of the other TZs remained stable. Therefore, the even-mode of TSSLR II determined the frequency position of TZ2, which was then able to adjust the skirt performance of the second passband. Except for the above-mentioned TZs in the center of the dual-passbands, two additional TZs were created in the upper stopband. In addition, TZ3 shifted to a higher frequency as the length of $L_{10}$ decreased, which resulted in changes in the selectivity of the right-hand skirt and the bandwidth of the second passband. When the length of $d_{1}$ was increased, which was equal to decreasing the length of the lower feedline $L_{14}$, TZ4 also shifted to a higher frequency. Thus, TZ4 at $8.0 \mathrm{GHz}$ could be controlled by managing the SLC between the lower feed-lines to improve the out-of-band rejection at high frequencies.

\section{Results and Discussion}

The proposed DBUWB BPF layout was shown in Fig. 9 and it was fabricated on Teflon substrate with the physical parameters shown in Tab. 1. The measured frequency response was found to match the results of the simulation, as plotted in Fig. 10. The 3-dB bandwidths of the dual passbands were measured to be $42 \%(1.7 \mathrm{GHz})$ 


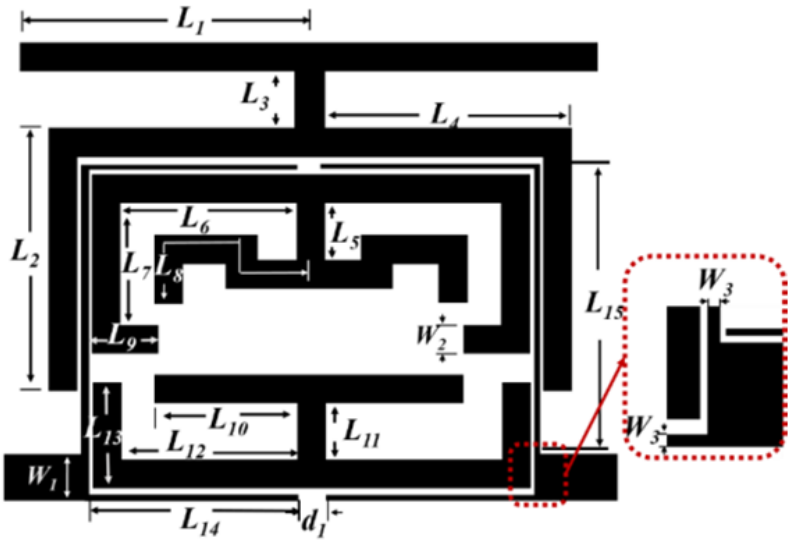

Fig. 9. Layout of the proposed DBUWB BPF.

\begin{tabular}{|c|c|c|c|c|c|c|}
\hline $\boldsymbol{L}_{\mathbf{1}}$ & $\boldsymbol{L}_{\mathbf{2}}$ & $\boldsymbol{L}_{\mathbf{3}}$ & $\boldsymbol{L}_{\mathbf{4}}$ & $\boldsymbol{L}_{\mathbf{5}}$ & $\boldsymbol{L}_{\mathbf{6}}$ & $\boldsymbol{L}_{\mathbf{7}}$ \\
\hline 10.6 & 7.7 & 1.7 & 9.6 & 1.7 & 8.0 & 4.7 \\
\hline $\boldsymbol{L}_{\mathbf{8}}$ & $\boldsymbol{L}_{\mathbf{9}}$ & $\boldsymbol{L}_{\mathbf{1 0}}$ & $\boldsymbol{L}_{\mathbf{1 1}}$ & $\boldsymbol{L}_{\mathbf{1 2}}$ & $\boldsymbol{L}_{\mathbf{1 3}}$ & $\boldsymbol{L}_{\mathbf{1 4}}$ \\
\hline 7.5 & 1.4 & 5.7 & 1.7 & 8.0 & 2.4 & 7.7 \\
\hline $\boldsymbol{L}_{\mathbf{1 5}}$ & $\boldsymbol{W}_{\mathbf{1}}$ & $\boldsymbol{W}_{\mathbf{2}}$ & $\boldsymbol{W}_{\mathbf{3}}$ & $\boldsymbol{d}_{\mathbf{1}}$ & & \\
\hline 8.6 & 1.5 & 1.0 & 0.2 & 1.6 & \multicolumn{3}{|c|}{ Unit: $\mathbf{m m}$} \\
\hline
\end{tabular}

Tab. 1. Physical parameters of the proposed DBUWB BPF.

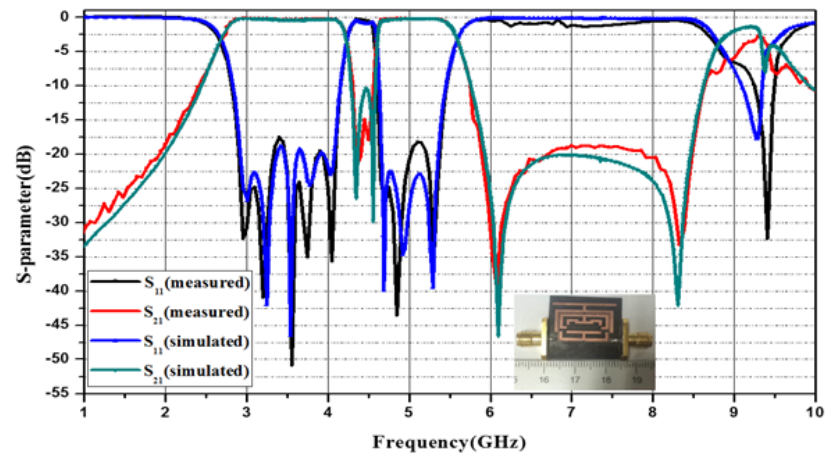

Fig. 10. Simulated and measured frequency responses of the proposed DBUWB BPF with the inset of the fabricated device.

and $22 \%(1.1 \mathrm{GHz})$, which were sufficient to satisfy the design specifications of the UWB. The center frequencies of the two passbands were $3.45 \mathrm{GHz}$ and $5.10 \mathrm{GHz}$, respectively, which correspond to the requirements for WiMAX $(3.5 \mathrm{GHz})$ and WLAN $(5.1 \mathrm{GHz})$ applications. The passband ripples in the first and second passband were $0.2 \mathrm{~dB}$ and $0.15 \mathrm{~dB}$, respectively, which demonstrated excellent in-band flatness. In addition, the maximum IL and minimum RL of the first and the second passbands were $0.8 / 17 \mathrm{~dB}$ and $0.9 / 18 \mathrm{~dB}$, respectively, which ensured reasonable in-band performance. The frequency response was below $-20 \mathrm{~dB}$ from $5.9 \mathrm{GHz}$ to $8.4 \mathrm{GHz}$, which showed an excellent out-of-band rejection. The other detailed measurement parameters are shown in Tab. 2. The overall size of the fabricated device was $21.2 \mathrm{~mm} \times 14.8 \mathrm{~mm}$, which was $0.35 \lambda_{\mathrm{g}} \times 0.25 \lambda_{\mathrm{g}}$ at the center frequency of $3.45 \mathrm{GHz}$. In addition, the comparison between the reference DWB BPFs and the proposed work were shown in Tab. 3, which proved the many benefits of the proposed DBUWB BPF in comparison, including superior bandwidths, in-band and out-of-band performances, as well as selectivity of four transitional band. All these performance superiorities were achieved by large amount RMs and TZs set at desired frequencies.

According to most of the published studies that use TSSLRs, RMs could only be generated at their inherent resonant frequencies. However, based on the results of the study reported in this paper, it was also possible to generate RMs based on the SQR of different resonators. In the passband frequency range, the BPF operated as a short-circuit between source and load because of the RMs. The corresponding strengths and sources of the RMs were changed by the different frequencies. If the resonators have similar electric lengths, then they would resonate simultaneously at the same frequency. This special phenomenon was referred to as $\mathrm{SQR}$ in this paper and led to the generation of new RMs with high RL. Therefore, not only were the basic RMs generated by resonators in the proposed design, but also additional RMs were generated by SQR to further improve the in-band performance.

\begin{tabular}{|c|c|c|c|c|}
\hline Passbands & \multicolumn{2}{|l|}{$1^{\text {st }}$ passband } & \multicolumn{2}{|c|}{$2^{\text {nd }}$ passband } \\
\hline Passbands (3 dB) & \multicolumn{2}{|c|}{$\begin{array}{c}2.6-4.3 \mathrm{GHz} \\
\left(f_{1}=3.45 \mathrm{GHz}\right)\end{array}$} & \multicolumn{2}{|c|}{$\begin{array}{c}4.6-5.6 \mathrm{GHz} \\
\left(f_{2}=5.10 \mathrm{GHz}\right)\end{array}$} \\
\hline Bandwidth/FBW & \multicolumn{2}{|l|}{$1.7 \mathrm{GHz} / 42 \%$} & \multicolumn{2}{|c|}{$1.1 \mathrm{GHz} / 22 \%$} \\
\hline Passband Ripple & \multicolumn{2}{|l|}{$0.20 \mathrm{~dB}$} & \multicolumn{2}{|r|}{$0.15 \mathrm{~dB}$} \\
\hline Maximum IL & \multicolumn{2}{|l|}{$0.8 \mathrm{~dB}$} & \multicolumn{2}{|r|}{$0.9 \mathrm{~dB}$} \\
\hline Minimum RL & \multicolumn{2}{|l|}{$17 \mathrm{~dB}$} & & $18 \mathrm{~dB}$ \\
\hline \multirow{2}{*}{$\begin{array}{c}\text { Out-of-band } \\
\text { Rejection }\end{array}$} & $\begin{array}{l}1^{\text {st }} \text { rejection } \\
\text { band }\end{array}$ & \multicolumn{2}{|c|}{$\begin{array}{l}2^{\text {nd }} \text { rejection } \\
\text { band }\end{array}$} & $\begin{array}{l}3^{\text {rd }} \text { rejection } \\
\text { band }\end{array}$ \\
\hline & $\begin{array}{c}\mathrm{S}_{21}<-20 \mathrm{~dB} \\
\text { at }<2 \mathrm{GHz}\end{array}$ & \multicolumn{2}{|c|}{$\begin{array}{c}\mathrm{S}_{21}<-10 \mathrm{~dB} \\
\text { at } 4.3-4.7 \mathrm{GHz}\end{array}$} & $\begin{array}{c}\mathrm{S}_{21}<-20 \mathrm{~dB} \\
\text { at } 5.9-8.4 \mathrm{GHz}\end{array}$ \\
\hline
\end{tabular}

Tab. 2. Measurement results of the proposed DBUWB BPF.

\begin{tabular}{|c|c|c|c|c|c|c|c|c|c|c|}
\hline & \multirow{2}{*}{$\begin{array}{c}\text { Center } \\
\text { frequency } \\
1^{\text {st }} / \mathbf{2}^{\text {nd }} \text { band } \\
(\mathbf{G H z})\end{array} \mid$} & \multicolumn{2}{|c|}{$\begin{array}{c}\text { Max IL/ Min } \\
\text { RL(dB) } \\
\end{array}$} & \multicolumn{2}{|c|}{ RMs } & \multicolumn{2}{|c|}{ Selectivity (dB per GHz) } & \multirow{2}{*}{\begin{tabular}{|c|} 
High freq. \\
out-of-band \\
rejection $<-20$ \\
dB $(\mathrm{GHz})$ \\
\end{tabular}} & \multirow{2}{*}{$\begin{array}{c}3 \mathrm{~dB} \\
\text { bandwidth of } \\
1^{\text {st }} / 2^{\text {nd }} \text { band } \\
(\mathrm{GHz})\end{array}$} & \multirow{2}{*}{$\begin{array}{c}\text { Overall size } \\
\left(\lambda_{\mathrm{g}}\right)\end{array}$} \\
\hline & & $1^{\text {st }}$ band & $2^{\text {nd }}$ band & $1^{\text {st }}$ band & $2^{\text {nd }}$ band & $\begin{array}{c}1^{\text {st }} \text { band } \\
\text { (up/down) }\end{array}$ & $\begin{array}{c}2^{\text {nd }} \text { band } \\
\text { (up/down) }\end{array}$ & & & \\
\hline Ref [3] & $2.60 / 5.30$ & $1.5 / 9$ & $1.5 / 15$ & 2 & 2 & $70 / 92$ & $77 / 70$ & $>1.2$ & $0.8 / 0.9$ & \\
\hline $\operatorname{Ref}[5]$ & $2.45 / 5.80$ & 1.8 & 3.0 & 2 & 2 & 108/104 & $90 / 156$ & $>1$ & 0.40 .4 & \\
\hline Ref [12] & $1.63 / 2.42$ & $0.86 / 15$ & $0.97 / 15$ & 2 & 2 & $60 / 83$ & $40 / 65$ & 0.5 & $0.5 / 0.7$ & $0.69 \times 0.31$ \\
\hline Ref [13] & $2.34 / 3.46$ & $0.84 / 17$ & $1.21 / 16$ & 3 & 3 & $45 / 150$ & $60 / 55.71$ & $>1.7$ & $0.6 / 0.8$ & $0.20 \times 0.21$ \\
\hline Ref [14] & $3.32 / 5.32$ & $0.62 / 17$ & $0.91 / 19$ & 2 & 3 & $26 / 210$ & $78 / 32$ & 2.7 & $0.8 / 1.1$ & $0.18 \times 0.40$ \\
\hline Ref [15] & $3.70 / 5.80$ & $0.70 / 40$ & $1.00 / 30$ & 1 & 1 & $50 / 175$ & $70 / 27$ & 3.5 & $1.5 / 1.1$ & $0.39 \times 0.25$ \\
\hline This work & $3.45 / 5.10$ & $0.8 / 17$ & $0.9 / 18$ & 5 & 3 & $22 / 122$ & $299 / 86$ & 2.3 & $1.7 / 1.1$ & $0.35 \times 0.25$ \\
\hline
\end{tabular}

Tab. 3. Comparison between the proposed DBUWB BPF and reference DWB BPF filters. 


\section{Conclusion}

A DBUWB BPF with eight RMs and four TZs was presented in this paper. By setting the width of the feedlines and the size of the coupling gaps, a strong coupling strength was achieved, which ensured high-quality signal coupling. The mechanism of operation of the RMs and TZs was analyzed by studying the current distributions and Sparameters. Similar electric lengths between the different resonators led to SQR that increased the numbers of RMs to improve the in-band performance. An excellent out-ofband rejection in the upper stopband was assured by the presence of the TZs generated by both the SLC of the feedlines and virtual ground effect of the loaded-stub. The center frequencies of the dual-band were adjusted to $3.45 \mathrm{GHz}$ and $5.1 \mathrm{GHz}$, which resulted in a filter that could be applied to DBUWB WiMAX and WLAN applications.

\section{Acknowledgments}

This work was supported by the National Research Foundation of Korea (NRF) grant funded by the Korean government (MSIP) No. 2014R1A1A1005901 and No. 2015R1D1A1A09057081. This work was also supported by a research grant from Kwangwoon University in 2017.

\section{References}

[1] ZHU, L., SUN, S., MENZEL, W. Ultra-wideband (UWB) bandpass filters using multiple-mode resonator. IEEE Microwave and Wireless Components Letters, 2005, vol. 15, no. 11, p. 796-798. DOI: 10.1109/LMWC.2005.859011

[2] CHEN, L. P., MA, Z., ANADA, T. Synthesis of ultra-wideband bandpass filter employing parallel-coupled stepped-impedance resonators. IEEE Microwave, Antennas and Propagation, 2007, vol. 2, no. 8, p. 766-772. DOI: 10.1049/iet-map:20070342

[3] TSAI, L. C., HUSE, C. W. Dual-band bandpass filters using equallength coupled-serial-shunted lines and Z-transform techniques. IEEE Transactions on Microwave Theory and Techniques, 2004, vol. 52, no. 4, p. 1111-1117. DOI: 10.1109/TMTT.2004.825680

[4] CHEN, X. P., WU, K., LI, Z. L. Dual-band and triple-band substrate integrated waveguide filters with Chebyshev and quasielliptic responses. IEEE Transactions on Microwave Theory and Techniques, 2007, vol. 55, no. 12, p. 2569-2578. DOI: 10.1109/TMTT.2007.909603

[5] KUO, J. T., YEH, T. H., YEH, C. C. Design of microstrip bandpass filter with a dual-passband response. IEEE Transactions on Microwave Theory and Techniques, 2005, vol. 53, no. 10, p. 1331-1337. DOI: 10.1109/TMTT.2005.845765

[6] CHEN, F. C., CHU, Q. X., TU, Z. H. Tri-band bandpass filter using stub loaded resonators. Electronics Letters, 2008, vol. 44, no. 12 , p. 747-749. DOI: 10.1049/el:20081054

[7] CHEN, C. Y., HSU, C. Y. A simple and effective method for microstrip dual-band filters design. IEEE Microwave and Wireless Components Letters, 2006, vol. 16, no. 3, p. 246-248. DOI: 10.1109/LMWC.2006.873584

[8] LI, Y., WANG, C., KIM, N. Y. Design of very compact bandpass filters based on differential transformers. IEEE Microwave and Wireless Components Letters, 2015, vol. 25, no.7, p. 28-30. DOI: 10.1109/LMWC.2015.2427660
[9] LI, Y., WANG, C., YAO, Z., et al. Very compact differential transformer-type bandpass filter with mixed coupled topology using integrated passive device technology. Microelectronics Journal, 2015, vol. 46, no. 12, p. 1459-1463. DOI: 10.1016/j.mejo.2015.06.022

[10] XU, K. D., ZHANG, Y. H., FAN, Y., et al. Novel circular dualmode filter with capacitive and inductive source-load coupling for multiple transmission zeros. Journal of Electromagnetic Waves and Applications, 2012, vol. 26, no. 13, p. 1675-1684. DOI: 10.1080/09205071.2012.708966

[11] ZHANG, X. Y., SHI, J., CHEN, J. X., et al. Dual-band bandpass filter design using a novel feed scheme. IEEE Microwave and Wireless Components Letters, 2009, vol. 19, no. 6, p. 401-403. DOI: 10.1109/LMWC.2009.2020009

[12] ZHOU, J. G., FENG, W. J., CHE, W. Q. Dual-wideband bandpass filter using $\mathrm{T}$-shaped structure based on transversal signalinteraction concepts. Electronics Letters, 2012, vol. 48, no. 24, p. 1539-1540. DOI: 10.1049/el.2012.3419

[13] LI, J., HUANG, S. S., WANG, H., et al. A novel compact dualwideband bandpass filter with multi-mode resonators. Progress in Electromagnetics Research Letters, 2015, vol. 51, p 79-85. DOI: 10.2528/PIERL14122302

[14] LI, J., HUANG, S. S., ZHAO, J. Z. Compact dual-wideband bandpass filter using a novel penta-mode resonator (PMR). IEEE Microwave and Wireless Components Letters, 2014, vol. 24, no. 10 , p. 668-670. DOI: 10.1109/LMWC.2014.2341014

[15] WU, Y. L., LIAO, C., XIONG, X. Z. A dual-wideband bandpass filter based on E-shaped microstrip SIR with improved upperstopband performance. Progress in Electromagnetics Research Letters, 2010, vol. 108, p. 141-153. DOI: 10.2528/PIER10071802

\section{About the Authors ...}

Jun-Ge LIANG born in Yantai, China, is currently working toward the Ph.D. degree at Kwangwoon University, Seoul, Korea. His research interests include RF devices design, electronic-material based sensors, and neuroscience research on brain network.

Cong WANG was born in Qingdao, China, in 1982. He received the B.S. degree in Automation Engineering from Qingdao Technological University, China in 2005, and the M.S. and Ph.D. degrees in Electronic Engineering from Kwangwoon University, Korea in 2008 and 2011, respectively. His work includes passive device modelling and design, and fabrication process development and optimization. He has also co-authored two books and published more than 200 papers in domestic and international journals and conferences. In addition, he has over 40 patents registered in Korea.

Nam-Young KIM received the M.S. and Ph.D. degrees in Electronic Engineering from the State University of New York (SUNY) at Buffalo, NY, USA, in 1991 and 1994, respectively, and the M.S. and Ph.D. degree in Theology from Midwest University, St. Louis, MO, USA, in 2004 and 2006, respectively. He leads the RFIC and compoundsemiconductor-related research group at Kwangwoon University. He has authored or co-authored 175 refereed journal papers, 28 books, and 343 refereed conference papers. In addition, he holds over 116 patents, including semiconductor design patents. 